\title{
An Abnormal Temperature Dependence of Alkylpyrazines' Retention in Reversed-Phase Liquid Chromatography
}

\author{
Kentaro Yogo,*,** Chiharu TaKemura,* Yoshihiro SaIto, ${ }^{* \dagger}$ and Kiyokatsu Jinno* \\ *Department of Environmental and Life Sciences, Toyohashi University of Technology, 1-1 Hibarigaoka, \\ Tempakucho, Toyohashi 441-8580, Japan \\ **Morinaga \& Co., Ltd., 2-2-1 Shimosueyoshi, Tsurumi, Yokohama 230-8504, Japan
}

\begin{abstract}
Retention behaviors of pyrazine and alkylpyrazines on various stationary phases in reversed-phase liquid chromatography were examined. An abnormal temperature effect on the retention of alkylpyrazines with a mobile phase consisting of acetonitrile and water was observed when changing the column temperature. On the other hand, no similar trend was found with a methanol-water mobile phase. For all the columns investigated in this work, the above tendency to the temperature-dependence was consistently observed, suggesting that the abnormal temperature effect on the retention of alkylpyrazines could be mainly induced by an acetonitrile-based mobile phase.
\end{abstract}

(Received August 23, 2011; Accepted October 4, 2011; Published December 10, 2011)

\section{Introduction}

Pyrazine and its derivatives could be regarded as an important group of compounds in the food industry, because of a significant contribution to a flavor formed in various roasted, toasted and heated foods. They are a common constituent of foods, and are generated primarily from a heat-induced condensation between amino acids and sugars through the Strecker degradation. ${ }^{1}$ Pyrazine derivatives exhibit a wide variety of aromas in food. For example, 2,5-dimethylpyrazine and tetramethylpyrazine are important flavor components in cocoa, ${ }^{2}$ and 2-ethenyl-3,5dimethylpyrazine and 2-ethenyl-3-ethyl-5-dimethylpyrazine are also important as earthy smelling compounds in coffee products. ${ }^{3}$ Development of an efficient separation and the subsequent determination of pyrazines could be, therefore, an essential procedure for precise quality control of these food products.

Liquid chromatography (LC) is one of the most widely used methods for the separation and isolation of various compounds, including pyrazines, because of the good separation performance and a wide availability. In terms of the separation mechanism in LC, however, we still have to do a lot of work for the comprehensive understanding because there are many parameters controlling the actual separation process of the analytes in LC. The retention and separation mechanism of aromatic compounds including a variety of polycyclic aromatic hydrocarbons (PAHs) and pyrazines have been studied. ${ }^{4-17}$

On the basis of actual retention measurements for pyrazines in several experimental conditions, a theoretical interpretation and the subsequent retention prediction were also studied in our previous work, ${ }^{17}$ where the retention prediction models for pyrazine and alkylpyrazines were developed using multiple linear regression (MLR) and artificial neural networks (ANNs). During the study, the retention of these compounds was carefully

† To whom correspondence should be addressed.

E-mail: saito@ens.tut.ac.jp determined in various conditions, including a variety of mobile phase conditions and column temperatures. The results suggested the existence of abnormal temperature-dependence of the retention of several alkylpyrazines on a typical octadecylsilica (ODS) stationary phase with a mobile phase containing acetonitrile $(\mathrm{ACN})$ as the organic component in reversed-phase (RP) conditions.

In this work, the temperature effect on the retention of pyrazines was further studied over the temperature range from 10 to $60^{\circ} \mathrm{C}$ using various types of alkyl-bonded stationary phases and mobile phase compositions in RPLC.

\section{Experimental}

\section{Chemicals and reagents}

All regents, solvents and sample solutes were of analytical grade and were used as purchased without further purification process. ACN, methanol $(\mathrm{MeOH})$, uracil, 2-methylpyrazine, 2,3-dimethylpyrazine, 2,3,5-trimethylpyrazine and 2,3,5,6-tetramethylpyrazine were purchased from Wako Pure Chemical Industries, Osaka, Japan and pyrazine was purchased from Tokyo Kasei Kogyo, Tokyo, Japan. The chemical structures of alkylpyrazines used in this work are shown in Fig. 1. Water was purified by a Milli-Q Water Purification System (Millipore, Tokyo, Japan).

\section{LC measurements}

Stock solutions $(1000 \mu \mathrm{g} / \mathrm{L})$ of the standards were prepared by dissolving in water or methanol, and were used for preparing standard working solutions in the actual chromatographic analysis, where these stock solutions were diluted with mobile phase to obtain a concentration of $20 \mu \mathrm{g} / \mathrm{L}$. For the dead time measurement, the peak of uracil added in all samples solutions was used.

The retention behavior of pyrazines was studied with the following commercially-available columns: Inertsil ODS-3 and 
<smiles>c1cnccn1</smiles>

(i) pyrazine<smiles>Cc1nccnc1C</smiles>

(iii) 2,3-dimethylpyrazine<smiles>Cc1cnccn1</smiles>

(ii) 2-methylpyrazine<smiles>Cc1cnc(C)c(C)n1</smiles>

(iv) 2,3,5-trimethylpyrazine<smiles>Cc1nc(C)c(C)nc1C</smiles>

(v) 2,3,5,6-tetramethylpyrazine

Fig. 1 Chemical structures of pyrazine and alkylpyrazines used in this work.

C8-3 columns (GL Sciences, Tokyo, Japan), Capcell Pak C18 UG120 and C1 UG120 columns (Shiseido, Japan), a Sunrise C28 column (ChromaNic Technologies, Osaka, Japan), where all the column dimensions and the particle sizes are the same: $150 \mathrm{~mm} \times 4.6 \mathrm{~mm}$ i.d. and $5 \mu \mathrm{m}$.

The LC system consisted of a PU-980 pump, a Model MD-910 photo diode array detector (Jasco, Tokyo, Japan) and a Model 7125 injector (Rheodyne, Cotati, CA) with a $20-\mu \mathrm{L}$ injection loop. Column temperature was mainly controlled by a Model CO-2060 column oven (Jasco, Japan), while a home-made water bath with a temperature-controlling system was employed for the chromatographic measurements at subambient temperatures, as described earlier.6, ${ }^{6,7}$ For the data collection and processing, Borwin Chromatography Data Processing Software (Jasco, Japan) running on a personal computer was used.

LC measurements were performed with $\mathrm{ACN} /$ water or $\mathrm{MeOH} /$ water mobile phase at a flow rate of $0.6 \mathrm{~mL} / \mathrm{min}$. An isocratic elution was employed in all the chromatographic runs. The mobile phase was equilibrated for at least $20 \mathrm{~min}$ prior to the measurements. The detection of pyrazines was done with the detector set at a wavelength of $270 \mathrm{~nm}$. Spectrum measurements to make sure of the identification of analytes were carried out, if necessary. Measurements were performed at least 5 times for each set of separation conditions.

\section{Results and Discussion}

The separation of a standard mixture containing five pyrazines (Fig. 1) was carried out on all the stationary phases described above at different column temperatures. Figure 2 shows typical chromatograms for the separation of pyrazines at two different column temperatures, at 30 and $60^{\circ} \mathrm{C}$, on an ODS phase. With $\mathrm{ACN}$ as a component of the mobile phase, all the retention factors for these alkylpyrazines were increasing when the column temperature was elevated, suggesting an abnormal temperature-dependence of the retention factor as found in other previous reports. ${ }^{18,19}$ On the contrary, when the column temperature was elevated with $\mathrm{MeOH}$-based mobile phases, all the retention factors were gradually decreased, as normally found in typical RPLC separation conditions. ${ }^{20-24}$ Similar trends were also observed for all other alkyl-bonded stationary phases

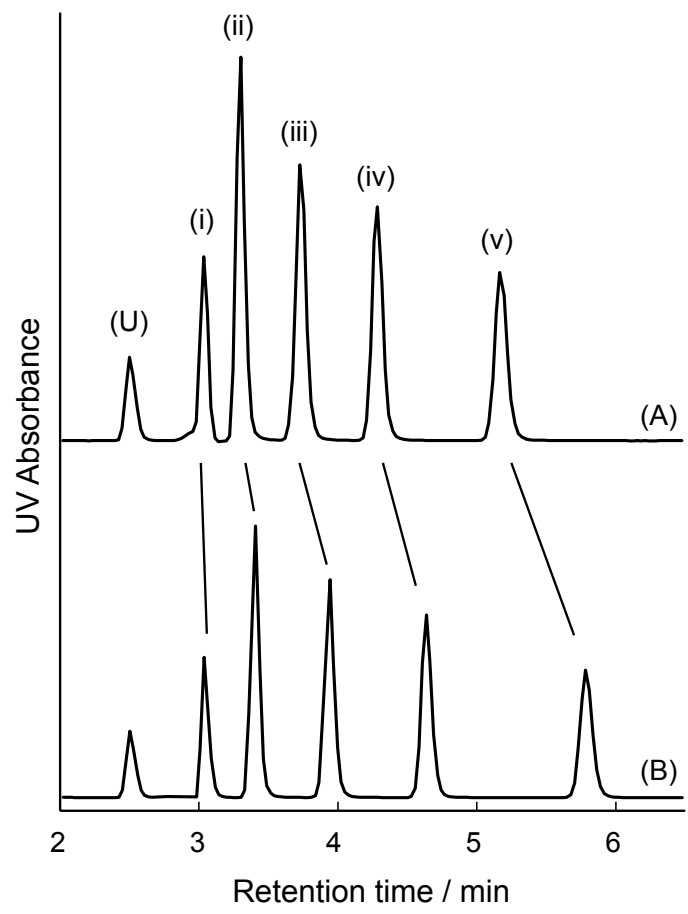

Fig. 2 Typical chromatograms for the separation of pyrazine and alkylpyrazines at different column temperatures: (A) 30 , (B) $60^{\circ} \mathrm{C}$. Other conditions: column, Inertsil ODS-3 $(5 \mu \mathrm{m}, 150 \mathrm{~mm} \times 4.6 \mathrm{~mm}$ i.d.); mobile phase, $\mathrm{ACN} / \mathrm{water}=30 / 70$; flowrate, $0.6 \mathrm{~mL} / \mathrm{min}$; detection, UV at $270 \mathrm{~nm}$. Peaks: (U) uracil, (i) pyrazine, (ii) 2-methylpyrazine, (iii) 2,3-dimethylpyrazine, (iv) 2,3,5-trimethylpyrazine, (v) 2,3,5,6-tetramethylpyrazine.

employed in this work.

For a better understanding of the abnormal temperature effect on the retention of pyrazines with $\mathrm{ACN}$ as the organic modifier in the mobile phase, van't Hoff plots were prepared as typically shown in Fig. 3. In RPLC with typical non-polar stationary phase, such as ODS phases, the retention can be expressed as the following equation:

$$
\ln k=-\Delta H / R T+\Delta S / R+\ln \Phi
$$

where $k$ is the solute retention factor, $\Delta H$ and $\Delta S$ are the enthalpy and the entropy of solute transfer from the mobile phase to the stationary phase, respectively. $R$ is the gas constant, $T$ the absolute temperature and $\Phi$ the volume phase ratio of the stationary phase and mobile phase. Equation (1) clearly shows a linear relationship between $\ln k$ and $1 / T$, known as the van't Hoff plot. Therefore, it is quite natural that a linear plot could be obtained in typical RPLC conditions, if the retention mechanism is constant over the temperature range studied. ${ }^{8-12}$

As can be found in the plots in Fig. 3, with a mobile phase consisting of $\mathrm{MeOH}$ and water, a good linear relationship was observed for all alkylpyrazines on both octyl- and octadecyl-bonded (i.e. ODS) stationary phases. Logarithmic retention factors for all the analytes are increasing linearly with the decrease of the column temperature as confirmed in most of the column temperature studies in RPLC. For all the measurements with ACN as the organic solvent in the mobile phase, however, the retention factors are logarithmically decreasing with the decrease of the column temperature, clearly showing an abnormal temperature effect on the retention with a mixture of $\mathrm{ACN} /$ water as the mobile phase. In addition, 
(A)
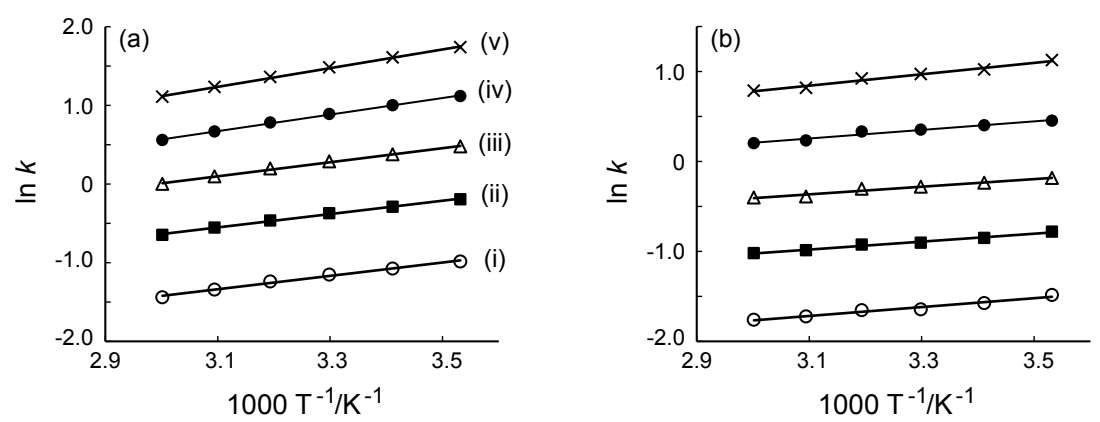

(B)
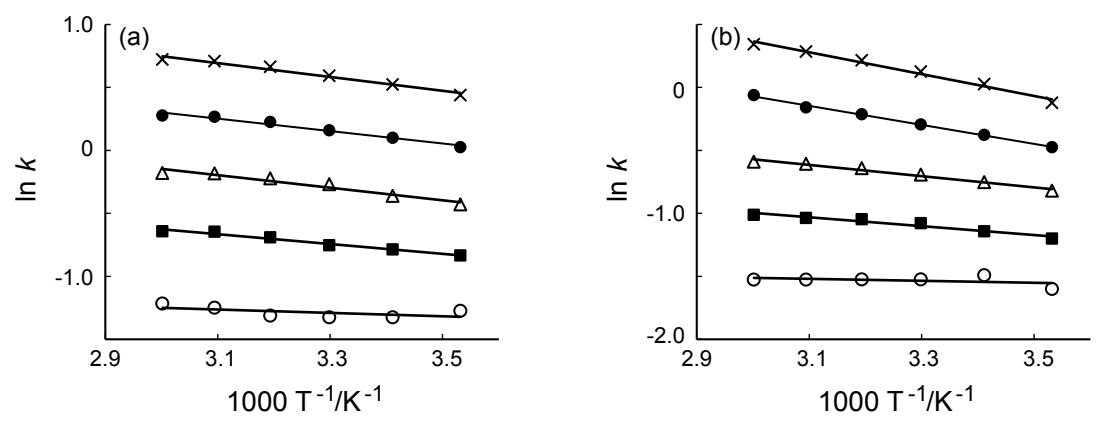

Fig. 3 van't Hoff plots for pyrazines with (A) $\mathrm{MeOH} /$ water $=40 / 60$ and (B) ACN/water $=30 / 70$ as the mobile phase on two types of bonded stationary phases. Stationary phase: (a) Inertsil ODS-3 $(5 \mu \mathrm{m}$, $150 \mathrm{~mm} \times 4.6 \mathrm{~mm}$ i.d.), (b) Inertsil C8-3 (5 $\mathrm{m}, 150 \mathrm{~mm} \times 4.6 \mathrm{~mm}$ i.d.). Other chromatographic conditions and the peak assignments are the same as in Fig. 2.

a similar trend was also observed for all other stationary phases, including $\mathrm{C} 1$ and $\mathrm{C} 28$ phases, studied in this work.

During the above temperature study, all the retention factors at a particular column temperature were measured at least 5 times, and no hysteretic effect was observed for the comparison of retention factors measured when the column temperature was sequentially increased or decreased to reach the desired temperature. As can be expected, for all the stationary phases studied, the retention of pyrazines was deceased with increasing the content of organic solvent, $\mathrm{MeOH}$ or $\mathrm{ACN}$, at constant column temperature, indicating that the dependence of the retention on the volume fraction of organic solvent in the mobile phase could be the same tendency as found in conventional RPLC separations. ${ }^{4,5}$

Table 1 summarizes the calculated enthalpy of solute transfer from the mobile phase to an ODS stationary phase. Although all the analytes showed negative enthalpy of transfer from mobile phase to stationary phase with $\mathrm{MeOH} /$ water as the mobile phase, as can be expected from Fig. 3, the values of enthalpy obtained with certain mobile phase compositions consisting of ACN and water are positive. In addition, when increasing the content of $\mathrm{ACN}$ in the mobile phase, the value of enthalpy will be decreased, showing a relatively more thermodynamically stable stay of pyrazines in the stationary phase. Similar studies were also carried out for pyridine and triazine, however, the behavior could be classified as a normal temperature dependence in conventional RPLC conditions. The logarithmic retention factors are increasing linearly with the decrease of the column temperature, and there were no observable differences in the temperature dependence of the retention between the mobile phases of $\mathrm{MeOH} /$ water and
Table 1 Standard enthalpy $\left(\mathrm{kJ} \mathrm{mol}^{-1}\right)$ of transfer for pyrazines from various mobile phases to the bonded phase of the ODS column

\begin{tabular}{crrrrrrrr}
\hline & \multicolumn{6}{c}{ Mobile phase } \\
\cline { 2 - 3 } Analyte $^{a}$ & \multicolumn{3}{c}{ MeOH/water } & & \multicolumn{3}{c}{ ACN/water } \\
\cline { 2 - 3 } \cline { 6 - 8 } & $30 / 70$ & $40 / 60$ & $50 / 50$ & & $30 / 70$ & $40 / 60$ & $50 / 50$ \\
\hline i & -4.66 & -4.07 & -3.99 & & 0.67 & -1.25 & -5.99 \\
ii & -4.49 & -3.66 & -2.24 & & 2.91 & -1.08 & -3.24 \\
iii & -5.57 & -3.49 & -2.49 & & 3.74 & 2.00 & -1.08 \\
iv & -5.99 & -3.91 & -2.58 & & 6.32 & 4.49 & 2.16 \\
v & -6.98 & -5.24 & -3.33 & & 7.23 & 6.15 & 3.82 \\
\hline
\end{tabular}

a. All the assignments are the same as in Fig. 1.

\section{ACN/water.}

Similar to the extensive studies on the retention behaviors of other aromatic compounds such as $\mathrm{PAHs},{ }^{4-7}$ more precise and comprehensive considerations, including theoretical interpretation of this phenomena, should be scheduled for the analysis of this unusual temperature dependence. At this stage, however, it can be said that the increased retention of pyrazines at elevated column temperature might be induced by the abnormal thermodynamic behavior during the partition between these stationary phases and the mobile phase containing ACN at a certain content. This abnormal temperature dependence might be useful for the development of a more efficient, but environmentally friendly, separation protocol of alkylpyrazines in RPLC. 


\section{Conclusions}

An abnormal temperature effect on the retention of pyrazines was confirmed with mobile phases consisting of $\mathrm{ACN}$ and water in this work. In terms of the dependence of the retention on the content of organic solvent in the mobile phase at constant temperature, all the pyrazines showed a normal behavior, as found in most RPLC conditions. The results suggest that more efficient separation of pyrazines could be developed by tuning up both the mobile phase composition and the column temperature, although further theoretical studies should be made to interpret the abnormal dependence of the pyrazines' retention on the column temperature with ACN/water as the mobile phase solvent. These studies including a more comprehensive survey using other types of organic solvents and the theoretical considerations are currently in progress in our laboratory.

\section{Acknowledgements}

A part of this research was financially supported by a Grant-in-Aid for Scientific Research from the Japan Society for the Promotion of Science. The authors would also like to express their gratitude for technical support from Dr. I. Ueta, Department of Applied Chemistry, University of Yamanashi. One of the authors, K. Y., also acknowledges Dr. M. Kamei, Mr. N. Ookawara, Mr. K. Sugiyama and all the members in the laboratory of food analysis (Morinaga \& Co., Ltd.) for their valuable help and suggestions.

\section{References}

1. T. B. Adams, J. Doull, V. J. Feron, J. I. Goodman, L. J. Marnett, I. C. Munro, P. M. Newberne, P. S. Portoghese, R. L. Smith, W. J. Waddell, and B. M. Wagner, Food Chem. Toxicol., 2002, 40, 429 .
2. G. A. R. Wood and R. A. Lass, "Cocoa", 1989, Wiley, New York.

3. M. Czerny, R. Wagner, and W. Grosch, J. Agric. Food Chem., 2008, 44, 3268.

4. L. C. Sander and S. A. Wise, Anal. Chem., 1984, 56, 504.

5. L. C. Sander and S. A. Wise, Anal. Chem., 1987, 59, 2309.

6. H. Ohta, Y. Saito, K. Jinno, J. J. Pesek, M. T. Matyska, Y.-L. Chen, and W. R. Biggs, Chromatographia, 1995, 40, 507.

7. H. Ohta, Y. Saito, N. Nagae, J. J. Pesek, M. T. Matyska, and K. Jinno, J. Chromatogr. A, 2000, 883, 55.

8. W. Melander, D. Campbell, and C. Horvath, J. Chromatogr., 1978, 158, 215.

9. G. Y. Vigh and Z. Varga-Puchony, J. Chromatogr., 1980, 196, 1.

10. L. C. Sander and L. R. Field, Anal. Chem., 1980, 52, 2009.

11. A. Tchapla, S. Heron, H. Colin, and G. Guiochon, Anal. Chem., 1988, 60, 1443.

12. L. A. Cole and J. G. Dorsey, Anal. Chem., 1992, 64, 1317.

13. Y. Saito, H. Ohta, and K. Jinno, Anal. Chem., 2004, 76, 267A.

14. K. Ban, Y. Saito, and K. Jinno, Anal. Sci., 2004, 20, 1403.

15. K. Ban, Y. Saito, and K. Jinno, Anal. Sci., 2005, 21, 397.

16. N. S. Quiming, N. L. Denola, A. B. Soliev, Y. Saito, and K. Jinno, Anal. Sci., 2008, 24, 139.

17. K. Yogo, N. S. Quiming, Y. Saito, and K. Jinno, Chromatographia, 2009, 70, 677.

18. R. Adlof and G. List, J. Chromatogr. A, 2004, 1046, 109.

19. N. Jinno, M. Murakami, M. Hashimoto, and K. Tsukagoshi, Anal. Sci., 2010, 26, 737.

20. D. V. McCalley, J. Chromatogr. A, 2000, 902, 311.

21. Y. Saito, H. Ohta, and K. Jinno, J. Sep. Sci., 2003, 26, 225.

22. C. B. Castells, L. G. Gagliardi, C. Rafols, M. Roses, and E. Bosch, J. Chromatogr. A, 2004, 1042, 23.

23. L. C. Sander, K. A. Lippa, and S. A. Wise, Anal. Bioanal. Chem., 2005, 382, 646.

24. M. Roses, X. Subirats, and E. Bosch, J. Chromatogr. A, 2009, 1216, 1756. 